\title{
Atomic scale understanding of the electronic structure of 5d-3d perovskite oxide heterostructures using STEM-EELS.
}

Sandhya Susarla ${ }^{1}$, Xiaoxi Huang ${ }^{2}$, Shehrin Sayed ${ }^{3}$, Lucas Caretta ${ }^{2}$, Hongrui Zhang ${ }^{2}$, Sayeef Salahuddin $^{3}$, Peter Ercius ${ }^{4}$ and Ramamoorthy Ramesh ${ }^{5}$

${ }^{1}$ National Center for Electron Microscopy, Lawrence Berkeley National Laboratory, Berkeley, CA 94720, USA, Berkeley, California, United States, ${ }^{2}$ Department of Materials Science and Engineering, University of California, Berkeley, CA 94720, USA, United States, ${ }^{3}$ Department of Electrical Engineering and Computer Science, University of California, Berkeley, CA 94720, USA, United States, ${ }^{4}$ Lawrence Berkeley National Laboratory, United States, ${ }^{5}$ University of California, Berkeley, Berkeley, California, United States

Creating abrupt heterostructures of materials with high spin-orbit coupling (SOC) such as topological insulators, heavy metals, 5d transition metal oxides etc. and ferromagnets such as NiFe, Permalloy, $\mathrm{LaMnO}_{3}$ etc. can result in high spin to charge conversion, useful for applications in spintronics. ${ }^{[1]}$ Most of these effects happen only at the interface, but the resolution offered by STEM-EELS provides the ability to study interfacial phenomena and the driving force behind charge to spin conversion. We studied $\mathrm{SrIrO}_{3} / \mathrm{La} 0.66 \mathrm{Sr} 0.33 \mathrm{MnO}_{3}$ (SIO/LSMO) as a model system to understand at atomic resolution the local electronic structure that produces high spin torque efficiency.

Epitaxial LSMO/SIO bilayers with varying SIO thickness were grown on $\mathrm{SrTiO}_{3}$ (STO) (0 0 1) substrates by high-pressure reflective high energy electron diffraction (RHEED)-assisted pulsed laser deposition. An atomic resolution HAADF-STEM image is displayed in Figure 1a. A sharp interface is evident from Figure1(a) with $\mathrm{MnO}_{2}$ termination in LSMO and SrO termination in SIO. The lattice parameters for various SIO thicknesses were extracted from HAADF-STEM images using Gaussian fitting of each atomic column. The LSMO out-of-plane (OOP) lattice parameter remains unchanged, but the OOP lattice parameter for SIO changes as a function of SIO thickness from $3.97 \AA$ for the 10 -unit cell (UC) SIO sample to $4.06 \AA$ for the 30 -UC SIO. We note that the SIO OOP lattice parameter $(3.97 \AA)$ in the $\operatorname{LSMO}(50$ $\mathrm{UC}) / \mathrm{SIO}(10 \mathrm{UC})$ is smaller than what has been reported for $30 \mathrm{UC}$ SIO grown on a bulk STO substrate (4.08 $\AA$ ).

Next, we correlate the electronic structure associated with this puzzling structural evolution by atomic resolution STEM-EELS (Figure 1b). EELS spectral maps were collected using a Gatan Continuum GIF with a K3 direct electron detector. The detector allows atomic mapping of the core-loss edges with high signal to noise ratio even with short dwell times ( $2 \mathrm{~ms} /$ spectrum). The EELS maps were de-noised using non-negative matrix factorization (NMF) based on Poissonian noise. We observe a sharp LSMO/SIO interface with no Mn/Ir interdiffusion, and a -1eV shift of the Mn L-edge in the 10UC SIO sample compared to the 30UC SIO and a reference spectrum ${ }^{[2]}$ (Figure 1c). These changes in the Mn L-edge point to the strong possibility of a charge transfer from Ir to $\mathrm{Mn}$. We also observe a corresponding change in the O K-edge from the SIO and LSMO layer (Figure 1d). The changes in the first $5 \mathrm{eV}$ of the O K-edge from the SIO side reflect changes in the electronic structure of the SIO layer, arising from changes in the Ir-O hybridization. Charge transfer can also be accompanied by orbital reconstruction in the strained SIO layer. ${ }^{[3,4]}$ Bulk SIO is semimetal with strong spin-orbit coupling (SOC), which is comparable to the crystal field splitting (CFS). ${ }^{[5]}$ It has been recently reported that CFS can dominate over SOC in strained iridate systems. ${ }^{[6]}$ Evidence for such an orbital re-construction due to CFS could be seen in $\mathrm{O}$ K edge in Figure 
1(d). In LSMO(50 UC)/SIO(10 UC) the density of states for Ir-O related levels are broader than that those in $\mathrm{LSMO}(50 \mathrm{UC}) / \mathrm{SIO}(30 \mathrm{UC})$. We believe that due to the dominance of CFS over the SOC, $\mathrm{S}=1$ state is stabilized in the LSMO (50 UC)/SIO(10 UC) as seen in nickelate/iridate systems. ${ }^{[4]}$ We hypothesize that the changes in $\mathrm{O} \mathrm{K}$-edge spectra of $\mathrm{SIO}$ are due to a combination of charge transfer and orbital reconstruction (Figure 2). We note that for $\operatorname{LSMO}(50 \mathrm{UC}) / \mathrm{SIO}(30 \mathrm{UC})$, where the charge transfer is negligible, there is no orbital reconstruction in Ir $5 \mathrm{~d}$ levels.

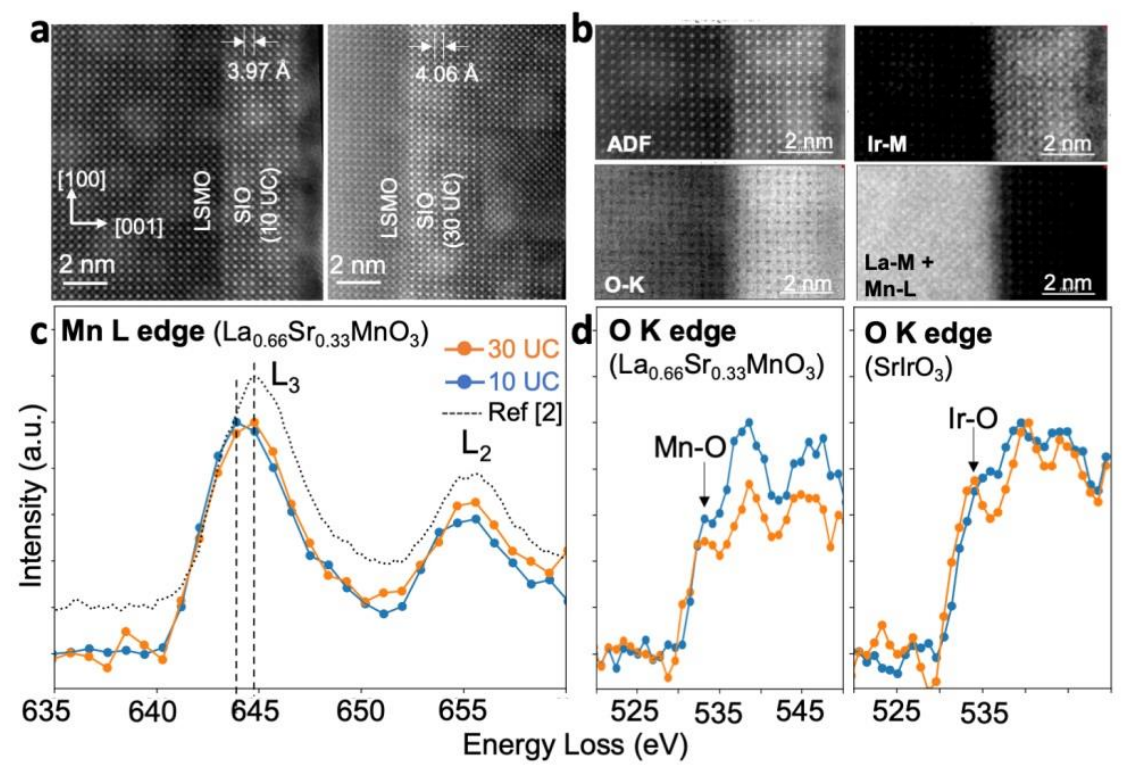

Figure 1. Figure 1: a) HAADF-STEM images of SIO/LSMO bilayers with 10UC and 30 UC SIO thickness. Overlayed are the OOP plane spacing in SIO extracted from lattice fitting. b) Atomic resolution STEM-EELS maps of LSMO/SIO displaying a sharp interface and no Mn/Ir interdiffusion. c) Average Mn L edge spectra extracted from the LSMO side of different LSMO/SIO heterostructures with a reference spectrum.[2] d) Average O K-edge extracted from LSMO and SIO sides showing different electronic structures depending on SIO thickness. 


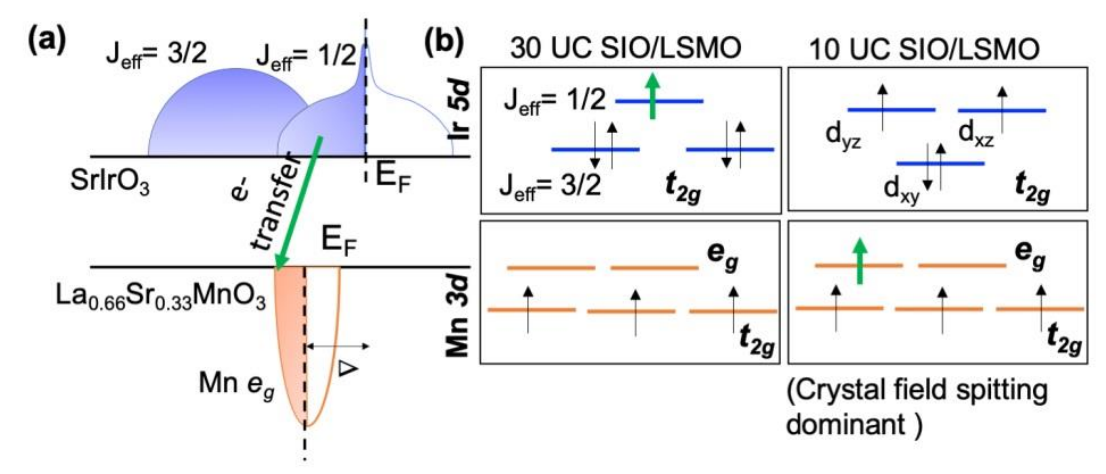

Figure 2. Figure 2: a) The electronic arrangement of LSMO/SIO showing the electron charge transfer from $\mathrm{Ir} 4+(\mathrm{SrIrO} 3)$ to $\mathrm{Mn} 4+(\mathrm{La} 0.7 \mathrm{Sr} 0.3 \mathrm{MnO} 3)$ b) Orbital reconstruction of $\mathrm{Mn} 3 \mathrm{~d}$ and Ir 5d levels for LSMO (50 UC)/SIO(10 UC) after the electron transfer. No electron transfer occurs for LSMO (50 UC)/SIO(30 $\mathrm{UC})$.

\section{References}

[1] W. Han, Y. Otani, S. Maekawa, npj Quantum Mater. 2018, 3, 1.

[2] P. Ewels, T. Sikora, V. Serin, C. P. Ewels, L. Lajaunie, Microsc. Microanal. 2016, 22, 717.

[3] D. Yi, J. Liu, S.-L. Hsu, L. Zhang, Y. Choi, J.-W. Kim, Z. Chen, J. D. Clarkson, C. R. Serrao, E. Arenholz, P. J. Ryan, H. Xu, R. J. Birgeneau, R. Ramesh, Proc. Natl. Acad. Sci. 2016, 113, 6397 LP.

[4] X. Liu, M. Kotiuga, H.-S. Kim, Y. Choi, Q. Zhang, Y. Cao, M. Kareev, F. Wen, B. Pal, J. W. Freeland, Proc. Natl. Acad. Sci. 2019, 116, 19863.

[5] M. A. Zeb, H.-Y. Kee, Phys. Rev. B 2012, 86, 85149.

[6] X. Liu, V. M. Katukuri, L. Hozoi, W.-G. Yin, M. P. M. Dean, M. H. Upton, J. Kim, D. Casa, A. Said, T. Gog, Phys. Rev. Lett. 2012, 109, 157401.

[7] The electron microscopy experiments were performed at the Molecular Foundry, Lawrence Berkeley National Laboratory, which is supported by the U.S. Department of Energy under contract no. DE-AC02-05CH11231. S.S. is supported by the DOE EFRC on Quantum Coherence. 